\title{
Effets du travail du sol sur le comportement chimique et biologique du sol et les conséquences sur le rendement d'un blé tendre (Triticum aestivum L.) dans la région « non chernozem » de la fédération de Russie.
}

\author{
Garané Ali ${ }^{1^{*}}$, Koussao Somé1, Traoré Mamoudou² and Sawadogo Mahamadou ${ }^{3}$

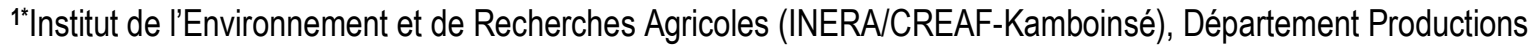 \\ Végétales, 01 BP 470 Ouagadougou 01, Burkina Faso. \\ 2Institut de l'Environnement et de Recherches Agricoles (INERA), CREAF-Kamboinsé, Département Gestion des \\ Ressources Naturelles/Systèmes de Production, 01 BP 476 Ouagadougou 01, Burkina Faso. \\ 3UFR/Sciences de la Vie et de la Terre. Université de Ouaga I Pr Joseph Ki-Zerbo, Laboratoire de Génétique et de \\ Biotechnologie Végétales, 03 BP 7021 Ouagadougou 03, Burkina Faso. \\ *Correspondance, courriel : ali_garane@yahoo.fr
}

Original submitted in on $3^{\text {rd }}$ February 2017. Published online at www.m.elewa.org on $31^{\text {st }}$ July 2017 https://dx.doi.org/10.4314/jab/v115i1.5

\section{RESUME}

Objectif : L'objectif de cette étude est de comparer les effets de six techniques culturales de mise en place du blé tendre sur certaines propriétés chimiques et biologiques du sol et les conséquences sur le rendement grain et ses composantes dans la région «non chernozem» en 7 ème années de rotation.

Méthodologie et résultats: Le dispositif expérimental utilisé est un Split plot à quatre répétitions comprenant chacune un témoin (labour conventionnel sur $20 \mathrm{~cm}$ ) et cinq traitements labours. II s'agit : du labour traditionnel sur $30 \mathrm{~cm}$, du travail minimum sur $8 \mathrm{~cm}$, l'alternance du labour conventionnel avec travail minimum, du labour conventionnel exécuté après un labour profond à la charrue à socs et enfin du labour conventionnel pratiqué après un labour profond l'aide au chisel anglais type Paraplay sur $30 \mathrm{~cm}$. Les résultats ont montré : pour le labour traditionnel continu (LT-27-30) et périodique (LC-20 après LT-27-30) des teneurs élevées en nitrate respectif de 28,5 et $27,7 \mathrm{mg} / \mathrm{kg}$ sur la couche $0-30 \mathrm{~cm}$. Pour les techniques minimales de travail (LM-8) et (LM-8/LC-20), ces quantités ont été supérieures dans les 10 premiers centimètres du sol respectif de 16,7 et $26,1 \mathrm{mg} / \mathrm{kg}$ et subissent une chute importante dans les horizons sous-jacents. Les quantités de phosphore et de potassium ont évolué sur la couche $0-20 \mathrm{~cm}$ de 21 à 27 $\mathrm{mg} / 100 \mathrm{~g}$ et de 14 à $15 \mathrm{mg} / 100 \mathrm{~g}$ respectivement. Le labour minimum sur cette couche a enregistré des concentrations beaucoup plus faible, soit $9,5 \mathrm{mg} / 100 \mathrm{~g}$ pour le phosphore et $10 \mathrm{mg} / 100 \mathrm{~g}$ pour potassium. Conclusion et application des résultats : Une étude économique est nécessaire pour se prononcer sur la technique à pratiquer. Cependant dans les conditions de nos essais, nous préconisons une alternance de la méthode traditionnelle avec labour profond avec le labour superficiel de $8 \mathrm{~cm}$. Le nombre d'années accordé à chaque type de labour dans la rotation doit être sensiblement égal pour l'obtention de meilleurs résultats.

Mots clés : Labour, blé tendre, paramètres chimiques, activité biologique, composantes, rendement 
Garane et al., J. Appl. Biosci. 2017 Effets du travail du sol sur le comportement chimique et biologique du sol et les conséquences sur le rendement d'un blé tendre dans la région « non chernozem », Russie.

Effects of soil tillage on the chemical and biological behavior of the soil and the consequences on the performance of soft wheat (Triticum aestivum L.) in the "non chernozem" region of the Russian Federation.

\section{ABSTRACT}

Objective: The purpose of our study was to compare the effect of six techniques routes of setting up of the soft wheat on some chemical and biologic properties of soil and the effect on grain yield and its components in the region non-chernozèm in 7th years of rotation"

Methodology and results: The experimental design used was a split plot design with six replications each comprising a control (classical plowing permanent $20 \mathrm{~cm}$ ) and five treatments plowings: traditional plowing $30 \mathrm{~cm}$, surface plowing $8 \mathrm{~cm}$, alternating tillage Classic $20 \mathrm{~cm}$ with shallow tillage, and finally conventional tillage executed after plowing the soil deep to $30 \mathrm{~cm}$ moldboard plow and chisel English in 7th year of rotation in the "non-chernozèm" area of Russia. The results showed that for the traditional (LT-27-30) and periodic (LC-20 after LT-27-30) plowing, high nitrate contents of 28.5 and $27.7 \mathrm{mg} / \mathrm{kg}$ respectively in the soil layer $0-30 \mathrm{~cm}$. For the minimum tillage techniques (LM-8) and (LM-8/LC-20), this variable was important in the first 10 centimeters of soil; 16.7 and $26.1 \mathrm{mg} / \mathrm{kg}$ respectively and very low in the underlying horizons. Amounts of phosphorus and potassium varied of 21 to $27 \mathrm{mg} / 100 \mathrm{~g}$ and of 14 to $15 \mathrm{mg} / 100 \mathrm{~g}$ in the layer $0-20 \mathrm{~cm}$ respectively. For these same variables, quantities evolved very closely, in the same order of 14 to $18 \mathrm{mg} / 100 \mathrm{~g}$ and 12 to $14 \mathrm{mg} / 100 \mathrm{~g}$ in the layer $20-30 \mathrm{~cm}$ respectively. Minimum tillage (LM-8) on this layer has registered much lower concentrations of $9.5 \mathrm{mg} / 100 \mathrm{~g}$ for phosphorus and $10 \mathrm{mg} / 100 \mathrm{~g}$ for potassium.

Conclusion and application of results: An economic study is necessary to decide on the technique to be practiced. However, under the conditions of these study tests, we recommend alternating traditional plowing $30 \mathrm{~cm}$ with surface plowing $8 \mathrm{~cm}$. The number of years for each type of tillage in the rotation must be substantially equal to obtain a better result

Key words: tillage, wheat, chemical properties, biological parameters, components, yield.

\section{INTRODUCTION}

Le labour, en enfouissant les résidus de cultures, crée des conditions favorables à la croissance microbienne et stimule l'activité de minéralisation des résidus de culture et de la matière organique du sol (Young et Ritz, 2000; Pekrun et al., 2003; Grigera et al., 2007). Cette technique a permis d'augmenter la productivité des cultures mais elle reste consommatrice de temps, de main d'œuvre, de puissance tractrice et d'énergie (Monnier, 1994). De plus, des problèmes de fertilité communs aux sols labourés demeurent l'émiettement, le tassement et la compaction des sols, l'appauvrissement en matière organique et l'érosion (Boiffin et al., 2001; Chervet et al., 2005; Mrabet, 2010) qui s'accompagne d'une réduction de la porosité du sol, de la circulation de l'oxygène et d'une augmentation du taux de saturation de la porosité en eau (Köller, 2003; Labreuche et al., 2007). Ces changements limitent le développement et l'activité des microorganismes aérobies et réduisent ainsi la minéralisation de la matière organique et de l'azote organique principal élément nutritif pour les végétaux. (Lipiec et Stepniewski, 1995). Pour toutes ces raisons, de plus en plus, sont adoptés des techniques qui couvrent une large gamme d'opérations allant du semis direct au travail du sol réduit sans retournement de la couche de sol avec un outil à dents ou à disques (Lal et al., 2007; Labreuche et al., 2007). L'objectif de ce travail, qui rentre dans le cadre d'un programme de recherche de «longue durée» est de comparer les effets de six techniques culturales de mise en place d'une céréale, blé tendre sur certaines propriétés chimiques et biologiques $\mathrm{du}$ sol et les conséquences son rendement grains et ses composantes dans la région «non chernozem» en 7ème années de rotation. 
MATERIEL ET METHODE

Le matériel végétal

Les caractéristiques essentielles de la variété sont : Le blé tendre (Tr. aestivum L. var. Eryhtrospermum Kôrn): Primorsks a été testée pour son adaptation à la culture intensive, ses rendements varient de 4 à 5 tha et 8,3 tha maximum. Semi-tardif, $70-90$ jours de par son cycle, il est très résistant contre les maladies cryptogamiques (fusarioses, Cercosporioses, septorioses) qui selon l'auteur Wiese (2001) sont responsables d'environ $5-40 \%$ des pertes de récoltes du blé.

Essai en station: Les expérimentations ont été conduites sur des parcelles expérimentales de l'Institut de Recherches Agronomiques dans la zone des terres non noires «non-chernozem» de la Fédération de la Russie. Sur la base d'essai «longue durée» sur l'étude des types de labours principaux sur la fertilité du sol, l'infestation de la culture par les adventices et le rendement du blé tendre de printemps dans une rotation de cultures suivantes: vesce et avoine, blé d'hiver; maïs pour l'ensilage, semis mixte orge et trèfle, trèfle en première année, Seigle d'hiver, et le blé de printemps. La parcelle expérimentale (dernovopodzolique), est un sol limoneux avec une teneur en particules argileuses $(<0,01 \mathrm{~mm})-40 \%$. La couche d'humus - $25-28 \mathrm{~cm}$. Avant l'installation de l'essai (2008), les caractéristiques agrochimiques ont été: acidité $(\mathrm{pH})$ sel - 5,8-6,3, acidité hydrolytique - 1,8-1,9 méq/100 $\mathrm{g}$ de sol, humus (par Tiourim) $-1,8-2,0 \%$, phosphore mobile (par Kirsanov) - 25,0 - 35,0 mg/100 $\mathrm{g}$ de terre et $\mathrm{K}_{2} \mathrm{O}$ (par Maslov) - $15,0-19,0 \mathrm{mg} / 100 \mathrm{~g}$ de terre. La densité du sol a été de $1,38-1,45 \mathrm{~g} / \mathrm{cm}^{3}$. En 2008, l'étude a porté sur l'effet de la profondeur et du type de labour principal sur certaines propriétés chimiques et biologiques du sol. Les conséquences sur le rendement grains et ses composantes du blé tendre suivant le dispositif expérimental suivant :

T1: Labour conventionnel: travail profond du sol effectué à l'aide d'une charrue à soc de profondeur avoisinant $20 \mathrm{~cm}$ (LC-20),

T2: Labour traditionnel: travail profond du sol a été effectué par une charrue à soc de profondeur avoisinant $30 \mathrm{~cm}$ (LP-27-30),

T3: Labour conventionnel suite a un labour profond de la charrue à socs sous l'orge d'hiver (CL-20 après LP27-30),

4: Travail minimum sur $8 \mathrm{~cm}$ pratiqué en alternance avec le labour conventionnel (TM-8/LC-20);

T5: Travail minimum continu sur $8 \mathrm{~cm}(\mathrm{TM}-8)$;

T6: Labour conventionnel pratiqué suite au labour du chisel sur $27-30 \mathrm{~cm}$ (LC-20 après L. chisel-27-30). Le schéma complet de l'expérience est donné dans le tableau 1.

Tableau 1 : Schéma de l'expérience.

\begin{tabular}{|c|c|c|c|c|c|c|c|}
\hline \multirow[t]{3}{*}{ Traitements } & \multicolumn{7}{|c|}{ Rotation des cultures } \\
\hline & 2002 & 2003 & 2004 & 2005 & 2006 & 2007 & 2008 \\
\hline & $\begin{array}{c}\text { Vesce + } \\
\text { avoine }\end{array}$ & $\begin{array}{c}\text { Blé } \\
\text { d'hiver }\end{array}$ & Maïs & $\begin{array}{c}\text { Orge + } \\
\text { trèfle }\end{array}$ & $\begin{array}{l}\text { Trèfle 1ère } \\
\text { année }\end{array}$ & Seigle d'hiver & $\begin{array}{c}\text { Blé de } \\
\text { printemps }\end{array}$ \\
\hline T1 & LC-20 & LC-20 & LC-20 & LC-20 & LC-20 & LC-20 & LC-20 \\
\hline T2 & TM-8 & LT-27-30 & LT -27-30 & LT -27-30 & LT -27-30 & LT -27-30 & LT $-27-30$ \\
\hline T3 & LT -27-30 & LC -20 & LC-20 & LC-20 & LC-20 & LT-27-30 & LC-20 \\
\hline T4 & LC-20 & LM-8 & LM-8 & LC-20 & LC-20 & TM-8 & LC-20 \\
\hline T5 & TM-8 & TM-8 & TM-8 & TM-8 & TM-8 & TM-8 & TM-8 \\
\hline T6 & $\begin{array}{c}\text { L. chisel- } \\
27-30\end{array}$ & LC-20 & LC-20 & LC-20 & LC-20 & $\begin{array}{c}\text { L. chisel- } \\
27-30\end{array}$ & LC-20 \\
\hline
\end{tabular}

LC -20: Labour classique (conventionnel) à $20 \mathrm{~cm}$ de profondeur ; TM- 8: Travail (Minimum) ou superficiel à $8 \mathrm{~cm}$ de profondeur ; LT-27-30 : Labour traditionnel à la charrue à soc à 27-30 cm de profondeur ; L. chisel -27-30 : Labour profond au chisel anglais type «Paraplay» à $27-30 \mathrm{~cm}$ de profondeur

En tout début septembre, le travail conventionnel et le labour traditionnel ont été réalisés à l'aide d'une charrue à socs de profondeurs 20 et $30 \mathrm{~cm}$ respectivement suivi d'un passage de cover-crop et un hersage, tandis que le labour profond sans retournement a été effectué à l'aide du chisel anglais type «Paraplay» de profondeur $30 \mathrm{~cm}$, suivi d'un passage de cover-crop et un hersage. Le cultivateur KPS-3 est utilisé pour le travail superficie du sol sur 8 $10 \mathrm{~cm}$, suivi d'un passage de cover-crop et un hersage. 
La parcelle expérimentale est à sa 7ème année d'installation. Elle est divisée en six grandes sous parcelles qui ont chacune subi un itinéraire technique différent. La parcelle élémentaire mesurait $312 \mathrm{~m}^{2}$ et la parcelle utile $200 \mathrm{~m}^{2}$ ( $25 \mathrm{~m} \times 8 \mathrm{~m}$ ). Les échantillons du sol analysés ont été prélevés dans les profils $0-10 \mathrm{~cm}$, $10-20 \mathrm{~cm}$ et $20-30 \mathrm{~cm}$ du sol. Un dispositif expérimental en bloc de Fisher randomisé à quatre (4) répétitions a été utilisé

Les analyses agrochimiques du sol: Les analyses agrochimiques du sol ont été conduites dans le laboratoire d'analyse de l'Institut de Recherches Agronomiques de la zone «non chernozem» de la Fédération de la Russie: humus (par Tiourim), nitrate d'azote (par Kfeldale), phosphore mobile (par Kirsanov), $\mathrm{K}_{2} \mathrm{O}$ (par Maslov). Les échantillons analysés ont été prélevé pendant la phase levée totale et maturité complète du blé dans les horizons 0-10, 10-20, 20-30 cm du sol.

Mesure de l'acidité hydrolytique du sol : II s'agit de l'acidité produite en traitant le sol avec une solution d'un sel de base forte et d'acide faible, généralement l'acétate de calcium (méthode Kappen). L'acidité hydrolytique peut servir à fixer avec une exactitude suffisante en pratique la quantité de chaux nécessaire à la neutralisation du sol. Dans ce but, le poids de chaux qui équivaut à l'acidité titrée est à multiplier par 1,5.

Les analyses microbiologiques du sol: Pour déterminer l'activité biologique du sol, la méthode de la

\section{RESULTATS}

Effets des techniques du travail du sol dans la régularisation du régime de nutrition : L'analyse de la teneur en nitrate d'azote (tableau 2) suggère deux conclusions importantes: Dans la première période de croissance du blé, la teneur en nitrate d'azote du sol a variée de 13,1 à $28,5 \mathrm{mg} / \mathrm{kg}$ sur la couche $0-30 \mathrm{~cm}$. Les traitements (LT-27-30) et (LC-20 après LT-27-30) avec labour traditionnel ont observé une tendance à la hausse de ce variable avec respectivement 28,5 et 27,7 $\mathrm{mg} / \mathrm{kg}$. Pour les techniques de travail minimum (LM-8) et (LM-8/LC-20), la teneur en nitrate a été supérieure dans les 10 premiers centimètres du sol $(16,7$ et 26,1 $\mathrm{mg} / \mathrm{kg}$ ) avec une chute importante dans les horizons sous-jacents. A la récolte du blé, les quantités de nitrate dans le sol ont chuté de 2,3 -9,4 mg/kg sous l'effet du lessivage (juillet) de la sècheresse (août) et en générale par la chute du procès de nitrification et leur absorption par les plantes en croissance pour la formation de la récolte. S'agissant des paramètres agrochimiques du sol, tels que la teneur en $\mathrm{P}_{2} \mathrm{O}_{5}, \mathrm{k}_{2} \mathrm{O}$ et décomposition de la cellulose par les microorganismes aérobics du sol par la minéralisation a été employée (Vostrova et Petrova, 1961). Elle consiste à déterminer la quantité résiduelle de cellulose non décomposée dans le sol en tenant compte de la variation du poids inhérent au sol des matériaux cellulosique. Des Plaques de verre $10 \times 50 \mathrm{~cm}$ de taille, sont recouvrent des toiles de lin d'un certain poids et insérées dans les horizons sol déterminés. Toutes les 4 semaines pour deux plaques sont enlevées, nettoyées du sol et traitées successivement avec $1 \%$ d'acide chlorhydrique, $1 \%$ de soude, de l'eau distillée et séchées. Le tissu est retiré de la plaque et pesé. Le changement de poids dans le tissu de lin caractérise la capacité cellulolytique du sol exprimée en grammes de poids sec de perte tissulaire ou en pourcentage du poids initial.

Le rendement en grains et ses composantes du blé : Le rendement en grains déterminé par battage des épis à partir des échantillons des bottillons fauchés manuellement sur un segment de rang de 3 mètres linéaires pour toutes les parcelles. Elles ont concerné le comptage du nombre des épis au mètre carré, Le poids de grains par mètre carré, le nombre de grains par plant et le rendement en grains déterminé par battage des épis. Le poids de mille grains est mesuré par comptage et pesée de 250 graines et sont déduits par calcul.

acidité (tableau 3), leurs valeurs ont été optimales au cours de l'essai (tableau 3). Ainsi, sur l'ensemble des traitements, la teneur du sol en phosphore mobile à variée de 21 à $27 \mathrm{mg} / 100 \mathrm{~g}$ en moyenne sur l'horizon $0-20 \mathrm{~cm}$. Sur horizon $20-30 \mathrm{~cm}$ a exception du travail minimum elle était de 14 à $18 \mathrm{mg} / 100 \mathrm{~g}$ pour l'ensemble des traitements. Le traitement (LM-8) avec travail du sol minimum enregistrait sur cette couche une teneur beaucoup plus faible, soit $9,5 \mathrm{mg} / 100 \mathrm{~g}$. L'évolution du potassium échangeable a été très étroite en moyenne sur le profil 0-20 cm (14-15 mg/100 g). Sur l'horizon $20-30 \mathrm{~cm}$ excepté le travail minimum (LM-8), la quantité de potassium échangeable a variée de 12 à $14 \mathrm{mg} / 100 \mathrm{~g}$ pour l'ensemble des traitements. Pour ce dernier, sa teneur $(10 \mathrm{mg} / 100 \mathrm{~g})$ quelque peu basse était la frontière entre moyenne et faible. Les types de labour principal du sol n'ont pas eu une influence substantielle sur l'acidité du sol et sur son évolution par horizon de labour. Dans l'ensemble des traitements les valeurs du pH ont varié de 6,1 à 6,7 , ce qui dans tout 
Garane et al., J. Appl. Biosci. 2017 Effets du travail du sol sur le comportement chimique et biologique du sol et les conséquences sur le rendement d'un blé tendre dans la région « non chernozem », Russie.

les cas constitue une neutralité. Certains traitements (LC-20, LT-27-30) ont enregistré une tendance légère à la baisse du $\mathrm{pH}$ avec la profondeur de labour. Cependant, aucune variation majeure de ce paramètre par traitement et par horizon de labour n'a été

observée. L'acidité hydrolytique a été faible pour l'ensemble les traitements et comprise entre 1,1 à 1,9 méq/100 g. Aucune variation logique de cette valeur par couche et en fonction des différents types de labour n'a été constatée.

Tableau 2 : Effet du mode de travail du sol sur le régime du nitrate du sol.

\begin{tabular}{|c|c|c|c|}
\hline \multirow[t]{2}{*}{ Traitements } & \multirow{2}{*}{$\begin{array}{l}\text { Horizons du sol } \\
(\mathrm{cm})\end{array}$} & \multicolumn{2}{|c|}{$\mathrm{NO}_{3}(\mathrm{mg} / \mathrm{kg})$} \\
\hline & & A la levée du blé & A la récolte du blé \\
\hline \multirow[t]{4}{*}{ T1 : LC-20 continu (témoin) } & $0-10$ & 16,3 & 8,2 \\
\hline & $10-20$ & 14,8 & 2,9 \\
\hline & $20-30$ & 8,15 & 2,1 \\
\hline & $0-30$ & 13,1 & 4,4 \\
\hline \multirow{4}{*}{ T2 : LT-27-30 continu } & $0-10$ & 36,4 & 17,4 \\
\hline & $10-20$ & 21,4 & 3,9 \\
\hline & $20-30$ & 27,6 & 2,8 \\
\hline & $0-30$ & 28,5 & 8 \\
\hline \multirow{4}{*}{ T3 : LC-20 (après LT- 27-30) } & $0-10$ & 31 & 12,6 \\
\hline & $10-20$ & 27,6 & 4,9 \\
\hline & $20-30$ & 24,6 & 2,7 \\
\hline & $0-30$ & 27,7 & 6,7 \\
\hline \multirow{4}{*}{ T4 : TM-8/LC-20 en alternance } & $0-10$ & 26,1 & 4,5 \\
\hline & $10-20$ & 24,6 & 1,8 \\
\hline & $20-30$ & 14,8 & 1,8 \\
\hline & $0-30$ & 21,8 & 2,7 \\
\hline \multirow{4}{*}{ T5 : TM - 8 continu } & $0-10$ & 16,7 & 3,5 \\
\hline & $10-20$ & 13,9 & 1,4 \\
\hline & $20-30$ & 10,8 & 2 \\
\hline & $0-30$ & 13,8 & 2,3 \\
\hline \multirow[t]{4}{*}{ T6 : LC-20 (après L.chisel 27-30) } & $0-10$ & 12,3 & 20,5 \\
\hline & $10-20$ & 21,4 & 4 \\
\hline & $20-30$ & 17 & 3,8 \\
\hline & $0-30$ & 16,9 & 9,4 \\
\hline
\end{tabular}

Tableau 3 : Éléments nutritifs et acidité du sol en fonction du mode de travail (à la récolte).

\begin{tabular}{|c|c|c|c|c|c|}
\hline \multirow[t]{2}{*}{ Traitements } & \multirow{2}{*}{$\begin{array}{l}\text { Horizons du } \\
\text { sol }(\mathrm{cm})\end{array}$} & $\mathrm{P}_{2} \mathrm{O}_{5}$ & $\mathrm{k}_{2} \mathrm{O}$ & \multirow[t]{2}{*}{$\mathrm{pH}_{\text {sel }}$} & \multirow{2}{*}{$\begin{array}{l}\text { Acidité hydrolytique } \\
\text { (méq/100 g sol) }\end{array}$} \\
\hline & & \multicolumn{2}{|c|}{$\mathrm{mg} / 100$ de sol } & & \\
\hline \multirow[t]{3}{*}{ T1 : LC-20 continu (témoin) } & $0-10$ & 27,2 & 15,4 & 6,74 & 1,25 \\
\hline & $10-20$ & 23,7 & 14,2 & 6,74 & 1,23 \\
\hline & $20-30$ & 18 & 12,8 & 6,11 & 1,6 \\
\hline \multirow{3}{*}{ T2 : LT-27-30 continu } & $0-10$ & 24 & 15,8 & 6,44 & 1,39 \\
\hline & $10-20$ & 20,2 & 13 & 6,7 & 1,12 \\
\hline & $20-30$ & 14 & 12,1 & 6,2 & 1,39 \\
\hline \multirow{3}{*}{ T3 : LC-20 (après LT- 27-30) } & $0-10$ & 29,7 & 15,4 & 6,61 & 1,87 \\
\hline & $10-20$ & 23,7 & 14,2 & 6,7 & 1,17 \\
\hline & $20-30$ & 18 & 12,8 & 6,6 & 1,28 \\
\hline \multirow{3}{*}{ T4 : TM-8/LC-20 en alternance } & $0-10$ & 26 & 16,3 & 6,66 & 1,39 \\
\hline & $10-20$ & 27 & 14,2 & 6,6 & 1,5 \\
\hline & $20-30$ & 18 & 13,4 & 6,5 & 1,45 \\
\hline \multirow[b]{2}{*}{ T5 : TM - 8 continu } & $0-10$ & 28,7 & 16,6 & 6,73 & 1,34 \\
\hline & $10-20$ & 22,2 & 13,5 & 6,61 & 1,44 \\
\hline
\end{tabular}


Garane et al., J. Appl. Biosci. 2017 Effets du travail du sol sur le comportement chimique et biologique du sol et les conséquences sur le rendement d'un blé tendre dans la région « non chernozem », Russie.

\begin{tabular}{|l|c|c|c|c|c|}
\hline & $20-30$ & 9,5 & 10 & 6,55 & 1,5 \\
\hline \multirow{3}{*}{ T6 : LC-20 (après L.chisel 27-30) } & $0-10$ & 23 & 13,8 & 6,52 & 1,5 \\
\cline { 2 - 5 } & $10-20$ & 18,6 & 13,4 & 6,63 & 1,39 \\
\cline { 2 - 5 } & $20-30$ & 17 & 13,7 & 6,37 & 1,5 \\
\hline
\end{tabular}

Effet des techniques du travail du sol sur l'activité biologique du sol: Les résultats d'analyse de l'activité biologique du sol ont montré (tableau 4) une plus forte activité des micro-organismes cellulolytiques sur la profondeur du labour $0-30 \mathrm{~cm}$ dans la technique de labour traditionnel (LT-27-30), soit 16,6\%. Aucune différence fondamentale n'a été observée entre le labour conventionnel continu (LC-20) et les labours profonds périodiques à la charrue 2 années sur 7 (LC20 après LT-27-30) ou au chisel 3 ans sur 7 (LC-20 après L.chisel-27-30) dans la rotation qui ont des pourcentages de décomposition de la cellulose respectifs de $14,3,14,8$ et $14,6 \%$. Au contraire les traitements (LM-8/LC-20) et (LM-8) ont enregistré des différences significatives. Dans ces derniers, l'activité des micro-organismes présente un gradient vertical où elle se concentre dans les premiers horizons de sol 0 $10 \mathrm{~cm}(18,7$ et $20 \%)$ et connait un affaiblissement brusque avec la profondeur. Une activité microbienne particulièrement faible a été observée sur la couche 20$30 \mathrm{~cm}$, où le taux de décomposition de la cellulose était de 4,9 et $4, \%$ respectivement, soit en moyenne 3 fois plus faible par rapport aux autres traitements.

Tableau 4 : Activité des microorganismes décomposeurs de la cellulose (cellulosiques) du sol.

\begin{tabular}{|c|c|c|c|}
\hline Traitements & Horizons du sol (cm) & $(\%)$ & $\begin{array}{l}\text { Pourcentage par } \\
\text { rapport au témoin(\%) }\end{array}$ \\
\hline \multirow[t]{4}{*}{ T1 : LC-20 continu (témoin) } & $0-10$ & 16,4 & - \\
\hline & $10-20$ & 13,9 & - \\
\hline & $20-30$ & 12,6 & - \\
\hline & $0-30$ & 14,3 & - \\
\hline \multirow{4}{*}{ T2 : LT-27-30 continu } & $0-10$ & 19,5 & 119 \\
\hline & $10-20$ & 16,9 & 122 \\
\hline & $20-30$ & 13,3 & 106 \\
\hline & $0-30$ & 16,6 & 116 \\
\hline \multirow{4}{*}{ T3 : LC-20 (après LT- 27-30) } & $0-10$ & 18,3 & 112 \\
\hline & $10-20$ & 14,6 & 105 \\
\hline & $20-30$ & 11,6 & 92 \\
\hline & $0-30$ & 14,8 & 103 \\
\hline \multirow{4}{*}{ T4 : TM-8/LC-20 en alternance } & $0-10$ & 18,7 & 114 \\
\hline & $10-20$ & 11,3 & 81 \\
\hline & $20-30$ & 4,9 & 39 \\
\hline & $0-30$ & 11,6 & 81 \\
\hline \multirow{4}{*}{ T5 : TM - 8 continu } & $0-10$ & 20,7 & 126 \\
\hline & $10-20$ & 8,3 & 60 \\
\hline & $20-30$ & 4 & 32 \\
\hline & $0-30$ & 11 & 77 \\
\hline \multirow[t]{4}{*}{ T6 : LC-20 (après L.chisel 27-30) } & $0-10$ & 17,2 & 105 \\
\hline & $10-20$ & 14,5 & 104 \\
\hline & $20-30$ & 12,1 & 96 \\
\hline & $0-30$ & 14,6 & 102 \\
\hline
\end{tabular}



du sol et les conséquences sur le rendement d'un blé tendre dans la région « non chernozem », Russie.

Effet des techniques du travail du sol sur les composantes de rendement: Le niveau des rendements est apprécié à priori par le niveau des différentes composantes constitutives du rendement, le peuplement épis, le nombre de grains par épi et le poids des grains (Gate, 1995). L'effet du mode de travail du sol était marqué sur les composantes de rendement au cours de l'essai (tableau 5). La densité des épis (épis $/ \mathrm{m}^{2}$ ) a varié significativement en fonction du mode de travail du sol. Le peuplement le plus élevé est compté sur les parcelles conduites en labour traditionnel continu, dépassant ainsi les traitements (LM-8/LC-20), (LM-8) et le témoin (LT-27-30) de $17 \%, 14 \%$ et $5 \%$ respectivement. Ces deux derniers traitements par ce variable ont cédé au témoin de $9 \%$ et $12 \%$ respectivement. Le nombre de grain a été moyen par plant avec cependant un effet majeur du mode de travail du sol sur ce paramètre. Le nombre le plus important est enregistré en labour traditionnel, soit des hausses respectifs de $26 \%$ et $23 \%$ par rapport au labour conventionnel et au travail minimum qui ont observé les chiffres les plus faibles. Le nombre de grain par plant a été déterminant dans le poids de grain par plant obtenu. Les traitements appliqués ont eu peu d'effet significatif sur la taille moyen du grain $(1,09-1,37$ g) quelque peu supérieur de $25 \%$ en labour traditionnel $(1,37 \mathrm{~g})$ en comparaison du témoin et du travail superficiel. Une position intermédiaire par ce variable mais aussi par la majorité des autres composantes est occupée par les traitements (LC-20 après L.chisel-2730) et (LC-20 après LT-27-30). Au delà, le poids de 1000 grain a été sensiblement identique pour l'ensemble des traitements de l'essai et varié de $39,3 \mathrm{~g}$ à $40,5 \mathrm{~g}$. Seule une légère baisse d'environ $3 \%$ est observée en travail superficiel continu par rapport aux autres traitements.

Tableau 5 : Effet du travail du sol sur le rendement grain et ses composantes

\begin{tabular}{|c|c|c|c|c|c|c|c|c|c|c|}
\hline \multirow[t]{2}{*}{ Traitements } & \multicolumn{7}{|c|}{ Composantes du rendement } & \multirow{2}{*}{$\begin{array}{c}\begin{array}{c}\text { Rende } \\
\text { ment } \\
\text { grain }\end{array} \\
\text { t/ha }\end{array}$} & \multicolumn{2}{|c|}{$\begin{array}{l}\text { Gain de } \\
\text { rendement } \\
\text { grain rapport } \\
\text { au témoin }\end{array}$} \\
\hline & $\begin{array}{l}\text { Densité } \\
\text { de peuple } \\
\text { ment } \\
\left(\text { plant } / \mathrm{m}^{2}\right)\end{array}$ & $\begin{array}{l}\text { Tailles- } \\
\text { utiles } \\
\text { (produc } \\
\text { tives) }\end{array}$ & Epis/m² & $\begin{array}{l}\text { Grains } \\
\text { par } \\
\text { plant } \\
\text { (grain/ } \\
\text { plant) }\end{array}$ & $\begin{array}{l}\text { Poids } \\
\text { grain/ } \\
\text { plant } \\
\text { (g) }\end{array}$ & $\begin{array}{l}\text { Poids } \\
\text { grain } \\
\text { par } \mathrm{m}^{2} \\
\left(\mathrm{~g} / \mathrm{m}^{2}\right)\end{array}$ & $\begin{array}{l}\text { Poids } \\
1000 \\
\text { grains } \\
\text { (g) }\end{array}$ & & t/ha & $\%$ \\
\hline $\begin{array}{l}\text { T1: LC-20 } \\
\text { continu } \\
\text { (témoin) }\end{array}$ & 304 & 1,29 & 392 & 27,1 & 1,09 & 331,36 & 40,5 & 3,34 & - & - \\
\hline $\begin{array}{l}\text { T2 : LT- } \\
27-30 \text { continu }\end{array}$ & 311 & 1,32 & 411 & 34,3 & 1,37 & 426,1 & 40,5 & 4,26 & $+0,92$ & $+27,5$ \\
\hline $\begin{array}{l}\text { T3 :LC-20 } \\
\text { (après LT-27- } \\
\text { 30) }\end{array}$ & 293 & 1,27 & 372 & 30,8 & 1,24 & 363,32 & 40,4 & 3,64 & $+0,3$ & +9 \\
\hline $\begin{array}{l}\text { T4 : TM-8/LC- } \\
20 \text { en } \\
\text { alternance }\end{array}$ & 274 & 1,28 & 351 & 29,2 & 1,17 & 320,58 & 40,4 & 3,22 & $-0,12$ & $-3,6$ \\
\hline $\begin{array}{l}\text { T5: TM- } 8 \\
\text { continu }\end{array}$ & 288 & 1,25 & 360 & 27,8 & 1,1 & 316,8 & 39,3 & 3,14 & $-0,2$ & -6 \\
\hline $\begin{array}{l}\text { T6 : LC-20 } \\
\text { (après } \\
\text { L.chisel } \\
27-30 \text { ) } \\
\end{array}$ & 293 & 1,3 & 381 & 31,2 & 1,27 & 372,11 & 40,6 & 3,71 & $+0,37$ & $+11,1$ \\
\hline $\mathrm{HCP}_{05}$ & & & & & & & & 0,288 & - & - \\
\hline
\end{tabular}

Effet des techniques du travail du sol sur le rendement grain : Le labour traditionnel (LT-27-30) a le plus amélioré son rendement en grains, dépassant le témoin (LC-20) de 0,92 t/ha (28\%) et le travail minimum continu (LM-8) de 1,12 t/ha (36\%). L'analyse de la variance entre ces traitements a révélé une différence très hautement significative, supérieure à $5 \%$ (tableau 5). Les traitements (LC-20 après L.chisel-27-30) et (LC- 

du sol et les conséquences sur le rendement d'un blé tendre dans la région « non chernozem », Russie.

20 après LT-27-30) avec des labours profonds périodiques de 3 et 2 années sur 7 dans la rotation ont cédé par leur rendement au labour traditionnel respectivement de $13 \%$ et $16 \%$. Cependant, ces derniers ont été supérieurs aux traitements (LC-20) de $11 \%$ et $9 \%$ et (LM- 8$)$ de $18 \%$ et $16 \%$ respectivement. Les différences de rendements des traitements (LC-20 après L.chisel-27-30) et (LC-20 après LT-27-30) ont été significatives par rapport aux labours traditionnel,

\section{DISCUSSION}

Effets des techniques du travail du sol dans la régularisation du régime de nutrition: Le nitrate constitue une des formes d'azote le plus active et plus disponible pour la plante. De plus, le taux de nitrate d'azote constitue une valeur qui varie très fortement aussi bien dans l'espace que dans le temps. Une tendance à la hausse de la teneur en nitrate d'azote est observée dans les traitements (LT-27-30) et (LC-20 après LT-27-30) avec labour traditionnel qui a enregistré 28,5 et $27,7 \mathrm{mg} / \mathrm{kg}$ respectivement. Au contraire, les traitements (LM-8) et (LM-8/LC-20) avec labour minimum ont enregistré des quantités du nitrate d'azote en général supérieures dans les 10 premiers centimètres du sol et décroissent fortement dans les horizons sous-jacents. Nos résultats sont similaires aux résultats (Al-Kaisi et Yin, 2005; Koch et Stockfisch, 2006; Gál et al., 2007; D'Haene et al., 2008) où ils notent que les concentrations du nitrate d'azote dans les systèmes qui incluent le travail minimum était en général supérieure dans les 10 premiers centimètres du sol par rapport à un sol labouré et décroissent fortement dans les horizons sous-jacents. Cependant, certains montrent que les techniques de travail du sol n'ont que peu d'effets sur ces stocks (Balesdent et al., 2000; D'Haene et al., 2008). En moyenne, le potassium mobile a connu une variation très étroite sur la couche $0-20 \mathrm{~cm}$, soit 14 à $15 \mathrm{mg} / 100 \mathrm{~g}$ de sol. Selon le recoupage des sols Dernovo-podzolitiques cet élément se situe à la frontière entre moyen et légèrement élevé. Cette valeur de potassium échangeable est jugée très satisfaisante si l'on considère que la majorité des sols Dernovo-podzolitiques ont une teneur moyenne ou faible de cet élément. Excepté le labour minimum continu (LM-8), la quantité de potassium (12 à 14 $\mathrm{mg} / 100 \mathrm{~g}$ ) a été moyenne sur la couche $20-30 \mathrm{~cm}$ et comprise entre moyenne et faible. Quant au phosphore mobile, en moyenne sa quantité a variée sur l'horizon $0-20 \mathrm{~cm}$ de 21 à $27 \mathrm{mg} / 100 \mathrm{~g}$. Selon le regroupement proposé dans agrochimie (1975), par la méthode de Kirçanova, une teneur en phosphore mobile comprise conventionnel et aux labours minimum (LM-8/LC-20) et (LM-8). Le traitement (LM-8/LC-20) qui alterne travail minimum et labour classique du sol a enregistré un rendement très proche du traitement (LM-8) avec travail minimum du sol continu, soit respectivement 3,22 et 3,14 t/ha. Cependant, comparé au témoin son rendement ce a été non significatif. Le plus faible rendement est enregistré avec le travail minimum permanent qui a été de $6 \%$ inférieur au témoin.

entre 15-25 mg/100 g est considérée comme élevée et au delà de $25 \mathrm{mg} / 100 \mathrm{~g}$ très élevée. Ces teneurs et répartitions du phosphore au cours de l'essai témoigne d'un apport suffisant de cet élément au sol. Le phosphore apporté par la fertilisation s'accumule plus sur les couches soumises au labour annuel ou périodique que sur l'horizon sous-jacent. Au cours du travail minimum, la couche $10-20 \mathrm{~cm}$ situé immédiatement sous l'horizon travaillé a enregistrée une teneur en phosphore plus élevée, tandis que sur la sous couche $20-30 \mathrm{~cm}$ plus profonde elle était fortement réduite. Selon Barber (1995), quelles que soient les techniques mises en œuvre, elles sont sans effet notable sur les teneurs en phosphore, en général très faibles, des couches de sols situées au dessous de la plus grande profondeur de travail en raison de la capacité qu'ont les racines à absorber du phosphore dans la solution du sol jusqu'à des concentrations de quelques $\mathrm{mg} / \mathrm{L}$. Les opérations culturales qui réalisent un retournement ou un malaxage du sol tendent à homogénéiser le phosphore dans le volume travaillé. Par contre celles qui n'engendrent pas de mélange des couches travaillées, conduisent à l'instauration d'un gradient de teneurs décroissantes avec la profondeur par l'enrichissement de la couche de surface qui interagit avec l'eau qui ruisselle (Sharpley, 2003).

Effet des techniques de travail du sol sur l'activité biologique du sol: Dans les profils de sols labourés, l'activité des microorganismes cellulolytique est plus forte sur la profondeur du labour $0-30 \mathrm{~cm}$ pour le labour traditionnel et $0-20 \mathrm{~cm}$ pour le labour conventionnel. Ces résultats confirment des travaux précédents qui montrent que la biomasse microbienne se développe préférentiellement au sein des horizons où les résidus de cultures sont enfouis (Andrade et al., 2003) pendant le labour traditionnel. L'ameublissement profond du sol conduit à une activation significative de l'activité des microorganismes décomposeurs de la cellulose et inversement son absence ou la compaction du sol supprime leur activité. Selon Berestetskiy (2001) le 
labour avec retournement augmentait très sensiblement la quantité de la flore microbienne, intensifiait la décomposition de la matière organique tandis que le coefficient d'humification était réduite. Le rythme de minéralisation augmentait ce qui réduisait la teneur en humus du sol et par conséquent sa fertilité lorsque les apports en matières organiques fraiches étaient insuffisants. Par contre, dans le traitement (LM-8/LC20) avec labour minimum périodique et plus particulièrement pour le traitement (LM-8) où le labour minimum est continu, l'activité des micro-organismes présente un gradient vertical et était plus importante fondamentalement sur les horizons supérieurs $0-10 \mathrm{~cm}$ et plus faible en profondeur. Ces résultats confirment ceux de nombreux auteurs qui ont également constaté que la biomasse microbienne et les activités de minéralisation de la matière organique étaient supérieures dans les premiers $\mathrm{cm}$ du sol dans les systèmes non labourés et diminuaient en profondeur (Andrade et al., 2003; Kaisi et Yin, 2005;; Gál et al., 2007; Koch et Stockfisch, 2006 ; D'Haene et al., 2008) suivant ainsi la répartition des résidus de culture dans le profil de sol.

Effet du travail du sol sur le rendement grain et ses composantes : L'analyse de la variance a révélé un effet systèmes de culture hautement significatif pour de rendement grain du labour traditionnel permanent (LT27-30). Le labour classique suite au chisel a été hautement significatif au témoin et aux traitements (LM8/LC-20) et (LM-8) avec labour minimum du sol. Le labour classique suite au labour traditionnel (LC-20 après LT-27-30) diffère du témoin approximativement par la plus petite valeur et d'une façon significative se démarque des traitements (LM-8/LC-20) et (LM-8). La baisse du rendement dans les traitements (LM-8/LC20) et (LM-8) par rapport au témoin a été non significative. Dans son ensemble, l'expérience a

\section{CONCLUSION}

Quelles que soient les techniques mises en œuvre, elles sont sans effet notable sur les teneurs en phosphore et de potassium mobiles en général très faibles, des couches de sols situées au dessous de la plus grande profondeur de travail. Seule le labour minimum continu observé une chute importante par rapport aux autres traitements des teneurs en phosphore et un léger recul de la quantité de potassium. L'activité des micro-organismes a été plus forte sur la profondeur du labour $0-30 \mathrm{~cm}$ pour le labour traditionnel et $0-20 \mathrm{~cm}$ pour le labour conventionne. Au contraire, les labours minimum continu et périodique montrée la supériorité de par son rendement du labour traditionnel permanent et l'existence de différences significatives par rapport au témoin des labours minimum travail périodique et permanent. Les résultats trouvés corroborent ceux de Triplett et Dick (2008) et de Camara et al. (2003). Effectivement, le blé cultivé sous système de conservation produit moins lorsqu'il est comparé au labour conventionnel. Le rendement plus élevé du labour traditionnel (LT-27-30) est le résultat d'un fort taux de levée, d'un peuplement plus important, d'un nombre d'épis et d'un poids de grains par mettre carré plus important dans ce traitement. Le travail du sol superficiel permanent a enregistré le plus faible rendement de $6 \%$ inférieur au témoin (LC-20). Cependant, cette différence reste en général insignifiante et ne peut pas être considérée comme significative. Les résultats similaires obtenus en Tunisie par M'hedhbi (1995) et Bouhedjba (1997), ont montré que la simplification du travail du sol réduit le rendement du blé par rapport à la méthode conventionnelle. De même, plusieurs années de travaux de recherches entamés au Maroc ont montré que le travail minimal ou l'absence de travail du sol n'entraîne quasiment aucune perte de rendement (Mrabet, 2001). Toutefois, les résultats obtenus au cours de la 5ème année de l'essai sont en faveur de la pratique en mode conventionnelle (El Bahri et al., 2000; Mrabet, 2001). Le poids de 1000 grains de blé a très peu évolué par traitement, l'analyse ressort que les rendements obtenus sont liés plus au nombre de grains formés par plant qu'au poids individuel d'un grain. Ces résultats corroborent avec ceux des auteurs Trottet et al. (2002); Sinclair and al. (2006) qui ont noté que le rendement était souvent très bien corrélé au nombre de grains formés alors qu'il est mal corrélé au poids individuel d'un grain.

ont présenté une activité biologique plus importante fondamentalement sur les horizons supérieurs $0-10 \mathrm{~cm}$ qui décroissent plus ou moins rapidement avec la profondeur de labour. Il a été ressorti une supériorité $\mathrm{du}$ labour profond permanent par le nombre d'épis au mettre carré, le nombre de grains par plant et l'effet négatif sur ces variables du travail du sol superficiel. Le labour traditionnel par son action plus profond du sol a permis un fort rendement. Aucune différence fondamentale n'a été observée entre le labour conventionnel et les labours minimum continus ou périodiques par le rendement. Le travail périodique 
profond à la charrue à socs ou au chisel a entrainé un

effet positif sur le rendement, cependant, son effet a été

\section{REFERENCES}

Al-Kaisi M. and Yin X. 2005. Tillage and crop residues effects on soil carbon and carbon dioxide emission in corn-soybean rotation. Journal of environnement quality, 34: 437- 445.

Andrade D.S., Colozzi-Filho A and Giller K.E. 2003. The soil microbial community and soil tillage. In A. El Titi (Ed.), Soil Tillage in Agroecosystems, CRC Press LLC Boca Raton, pp. 51-81.

Balesdent J.C. Chenu et Balabane M. 2000. Relationship of soil organic matter dynamics to physical protection and tillage. Soil \& Tillage Research, 53: 215-230.

Barber S.A. 1995. Soil Nutrient bioavailability. A mechanistic approach. John Wiley and sons, p. 414.

Berestetskiy O.A. 2001. Sol et les processus microbiologiques dans la rotation / Théorie et pratique de l'amélioration de la fertilité des sols. Moscou, pp. 27-29.

Boiffin J., Malezieux E. \& Picard D. 2001. Cropping systems for the future. In Nösberger J. Geiger, H. H., Struik, P. C. (eds.), Crop Science, pp 261-280.

Bouhejba A. 1997. Études comparatives des effets des différents modes de travail du sol sous des conditions semi arides sur l'élaboration du rendement du blé tendre. Mémoire de fin d'étude du cycle spécialisation INA Tunisie.

Carama K.M., Payne W.A. et Rasmussen P.E. 2003. Long-term effects of tillage, nitrogen and rainfall on winter wheat yields in the Pacific Northwest. European Journal of Agronomy, 95: 828-835.

Chervet A., Ramseier L., Sturny W. G. \& Tschannen S. 2005. Comparaison du semis direct et du labour pendant 10 ans. Revue suisse Agric. 37 (6): 249-256.

D'Haene K., Vandenbruwane J., De Neve S., Gabriels D., Salomez J. and Hofman G. 2008. The effect of reduced tillage on nitrogen dynamics in silt loam soils. Agron. J., 28: 449-460.

Gál A., Vyn T.J., Michéli E., Kladivko E.J. and McFee W.W. 2007. Soil carbon and nitrogen accumulation with long-term no-till versus moldboard plowing overestimated with tilled- moindre du faite de la périodicité du labour.

zone sampling depths. Soil and Tillage Research, 96:42-51.

Gate P. 1995. Écophysiologie du blé. Ed. Lavoisier, Paris, pp. 78-81.

Grigera M.S., Drijber R.A. et Wienhold B.J. 2007. Redistribution of crop residues during row cultivation creates a biologically enhanced environment for soil microorganisms. Soil and Tillage Research 94:550-554.

Koch H.J. et Stockfisch N. 2006. Loss of soil organic matter upon ploughing under a loess soil after several years of conservation tillage. Soil and Tillage Research 86:73-83.

Köller K. 2003. "Techniques of Soil Tillage", In A. El Titi, ed. Soil Tillage in Agro écosystème CRC Press LLC, Boca Raton, pp. 1-25.

Labreuche J., Viloingt T., Caboulet D., Daouze J.P., Duval R., Ganteil A., Jouy L., Quere L., Boizard H.et Roger-Estrade J. 2007. "Evaluation des impacts environnementaux des Techniques Culturales Sans labour (TCSL) en France". Partie I: La pratique des TCSL en France, Ademe.

Lal R., Reicosky D.C. and Hanson J.D. 2007. "Evolution of the plow over 10,000 years and the rationale

for no- till farming". Soil and Tillage Research, 93: 1-12.

Lipiec J. et Stepniewski W. 1995. Effects of soil compaction and tillage systems on uptake and lose of nutriments. Soil \& Tillage Research, 35:37-52.

M'Hedhbi K. 1995. Effet des outils de travail du sol et de semis sur le rendement des céréales cultivés en sec. Dans: Actes de la 27ème journée nationale sur les acquis de la recherche agronomique, halieutique et vétérinaire en Tunisie, 1: 36-45.

Mrabet R. 2000. Differential response of wheat to tillage management system under continues cropping in a semi-arid area of Morocco. Dans: Field crops research, 66:165-174.

Mrabet R. 2010. Climate change and carbon sequestration in the Mediterranean basin: contribution of

no tillage systems. Les actes des $4 e$ Rencontres Méditerranéennes du Semis Direct. Sétif 
Algérie $3,4,5$ mai $2010 . \mathrm{N}^{\circ}$ spécial revue Recherche agronomique. Pp : 106-126.

Pekrun C., Kaul H.P. et Claupein W. 2003. Soil tillage for sustainable nutrient management. In El Titi, A.

(ed.). Soil tillage in agroecosystems, CRC Press, NewYork (USA), pp. $83-113$.

Sharpley A.N. 2003. Soil mixing to decrease surface stratification of phosphorus in manure soils Journal of Environmental quality, 32: 13751384.

Sinclair T.R. et Jamieson P.D. 2006. "Grain number, wheat yield, and bottling beer an analysis, Field" Crops Research, 98: 60 - 67.

Smirnova P.M. et Petersburg's A.V. 1975. Agrochimie. Moscou, p. 512.
Triplett G.B. et Dick W.A. 2008. No-tillage crop production: A revolution in agriculture! Agron. J., $100: 153-165$.

Trottet M. et Doussinault G. 2002. "Analyse du progrès génétique chez le blé tendre au cours du XXème siècle". Le Sélectionneur Français, 53: $3-13$.

Vostrova I.S. et Petrova A. 1961. Différentes méthodes de détermination de l'activité biologique du sol. Microbiologie 30 (4): 665-672

Wiese M.V. 2001. Compendium of wheat diseases. The American Phytopathological Society p.106.

Young I.M. et Ritz K. 2000. Tillage, habitat space and function of soil microbes. Soil and Tillage. Research, 53:201-213. 